\title{
Research on DBN-based Evaluation of Distribution Network Reliability
}

\author{
Zhang Kaiyu ${ }^{1}$, Shi Shanshan ${ }^{1}$, Liu Shu ${ }^{1}$, Wan Junjie ${ }^{2}$, Ren Lijia ${ }^{2, *}$ \\ ${ }^{1}$ State Grid Shanghai Electric Power Research Institute, Shanghai, China \\ ${ }^{2}$ Shanghai University of Engineering Science, Shanghai, China
}

\begin{abstract}
In order to accurately and efficiently analyze the reliability of distribution network, this paper proposes a method of analyzing the reliability of distribution network based on a deep belief network. The Deep Belief Network (DBN) is composed of limiting Boltzmann machine layer-by-layer stacking. It has a strong advantage of automatic feature extraction, which overcomes the shortcomings of traditional neural networks in extracting data features. The entire training process of DBN can be roughly divided into two stages: pre-training and fine-tuning.First of all, the pre-training of the DBN model is realized by training the Restricted Boltzmann Machine (RBM) layer by layer, then the BP algorithm is used for reverse fine-tuning to complete the training process of the entire network. finally, the reliability analysis of distribution network is performed by the trained DBN. Compared with the BP neural network method and the traditional Monte Carlo simulation method, it is verified that the proposed model of distribution network reliability analysis has high accuracy.
\end{abstract}

\section{Introduction}

The distribution network is an important part of the power system, and its wide range and complex structure have an important impact on reliability. The safe and reliable power supply of the distribution network is related to the requirements of residents, the benefits of power enterprises, and the development of social economy, so it is particularly important to improve the reliability of the distribution network. The evaluation of distribution network reliability is a basic method to analyze the weaknesses of the distribution network structure and ensure the safe operation of the power system, whcih has important social significance and economic value for in-depth research on the reliability of the distribution network. Therefore, it is of great significance to study fast and accurate methods for distribution network reliability analysis [1].

The methods of distribution network reliability analysis usually include three types: simulation method, analytical method and artificial intelligence method. Among them, the most commonly used simulation method is the Monte Carlo method. The calculation accuracy of the Monte Carlo method is closely related to the number of samples. Given such features as large amount of calculation and long calculation time, Monte Carlo method is difficult to meet the needs of online analysis [2]. Analytical methods generally include state enumeration method [3], failure mode impact analysis method [4] and minimum path method [5]. Among them, the method of failure mode impact analysis is commonly used. However, as the complexity of the network structure increases, the difficulty of its reliability analysis also increases, resulting in a large amount of calculation and slow speed. The neural network method is the most common in artificial intelligence algorithms. The neural network algorithm analyzes based on historical sample data, and its adaptive ability is strong. After training, the reliability analysis time and calculation amount are reduced, but the calculation accuracy needs to be improved [6]. It is easier to estabilsh a model when using neural networks to evaluate the reliability of the distribution network. The solution and evaluation can be obtained by neural network if the network structure of the distribution network appropriately transformed. Reference [7] combined Monte Carlo method with artificial neural network and applied it in the reliability evaluation process to calculate the index of distribution network reliability. Reference [8] considered the relevant influencing factors that affect the reliability of the distribution network, and applied the BP neural network to the evaluation of the distribution network reliability. Reference [9] applied the radial basis function neural network to predict the reliability of distribution network components by fitting the original data to evaluate the reliability of the distribution network. Reference [10] combined fuzzy theory and neural network to evaluate the reliability of each load point index of the distribution network. Reference [11] divided the distribution network into several sub-networks, and used artificial neural networks to train and test each sub-network. This method can meet requirements of various types of distribution networks and has a strong ability of adaption. Reference [12] proposed a three-layer artificial neural

Corresponding author: rlijia@126.com 
network to train the historical reliability data of the distribution network and obtained the reliability index of the distribution network.

In recent years, many scholars have introduced artificial intelligence into the research of power systems. Artificial intelligence methods for distribution network reliability analysis include deep learning algorithm [13], genetic algorithm [14], particle swarm optimization algorithm [15], etc. Deep learning algorithm is the most commonly used method in artificial intelligence.

Deep neural network model has the structure feature of hidden layers stacked one by one, and each layer extracts different data features as the input of the next layer. Through the nonlinear relationship between input and output, low-level features can be combined with high-level abstract representations. This paper proposes a model of distribution network reliability analysis based on deep belief network. This model not only makes up for the shortcomings of neural network in reliability analysis in the past, but also solves simulation method and analytical method's problem of large amount of calculation, time-consuming, and impossibility to meet the needs of online analysis. The deep belief network used has multiple hidden layers, which can contain more information about the distribution network and obtain deeper essential characteristics of sample data. Using the trained deep learning network to analyze the reliability of the distribution network, the speed is fast and the error of the result obtained is small. This research can provide a reasonable basis for the planning and operation of the distribution network, and also has a high reference value for the cost-benefit analysis of the distribution network.

\section{DBN model}

DBN is a new machine learning algorithm, a deep neural network composed of multiple stacked restricted Boltzmann machines (RBM) and single-layer classifiers, using unsupervised greedy layer-by-layer pre-training to generate initial spatial distribution of weights and threshold values has great advantages in dealing with non-linear problems. A typical RBM consists of a visible layer and a hidden layer. The neurons between the visible layer and the hidden layer are connected to each other, and the neurons within each layer remain independent.

RBM is based on the concept of energy model. The joint energy function of visible layer neurons and hidden layer neurons is:

$$
E(v, h \mid \theta)=-\sum_{i=1}^{n} v_{1} a_{i}-\sum_{j=1}^{m} h_{j} b_{j}-\sum_{j=1}^{n} \sum_{j=1}^{m} v_{i} w_{i j} h_{j}
$$

In the formula, $\theta=\left\{a_{i}, b_{j}, w_{i j}\right\} ; a_{i}$ is the bias of the visible layer neuron; $b_{j}$ is the bias of the hidden layer neuron; $w_{i j}$ is the weight of connection between the visible layer and the hidden layer.

According to the energy function, the probability of connection of neurons in the visible and hidden layers can be obtained:

$$
P(v, h \mid \theta)=\frac{1}{z(\theta)} e^{-E(v, h \mid \theta)}
$$

Given that the state of neurons in the visible layer and the state of neurons in the hidden layer satisfy the condition of independence, the probability of activation of the $\mathrm{j}$-th neuron state in the hidden layer is:

$$
p\left(h_{j}=1 \mid v\right)=f\left(\sum_{i} v_{i} w_{i j}+b_{j}\right)
$$

In the same way, given the hidden layer neuron state, the activation probability of the i-th visible layer neuron state is:

$$
\begin{aligned}
& \qquad p\left(v_{i}=1 \mid h\right)=f\left(\sum_{i} h_{j} w_{i j}+a_{i}\right) \\
& \text { In the formula, } f(x)=\frac{1}{1+e^{-x}}
\end{aligned}
$$

RBM training adopts the contrast divergence method, reconstructs the input sample according to the data characteristics, compares it with the original sample, and adjusts the parameter $\theta$ with the maximum likelihood function as the target. $\theta$ can be obtained by the log likelihood function:

$$
L(\theta)=\sum_{n=1}^{N} p\left(v^{n}, h\right)
$$

The DBN structure is shown in Figure 1. It is composed of multiple RBMs, and the top layer is a BP neural network. The DBN training process is divided into two steps, pre-training and fine-tuning. Firstly, the unsupervised layer-by-layer pre-training method is used to pre-train each layer of RBM from bottom to top, and the output of the previous layer of RBM is used as the input of the next layer of RBM, so that each layer can learn the features of the previous layer and obtain the initial weight. Finally, the top-level BP network is used to perform top-down supervised learning to reversely fine-tune the network, thereby further optimizing network parameters. After pre-training and fine-tuning, the training process of the deep belief network is completed.

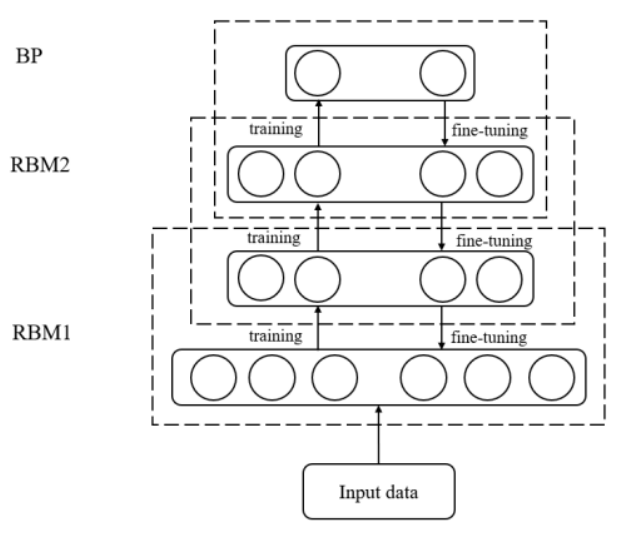

Fig. 1. DBN structure

\section{Steps of distribution network reliability analysis}

After analyzing the relevant factors affecting power supply reliability of the distribution network, this paper chooses the following: (1) the topology of the distribution network; (2) the line parameters of the distribution network (line model, line length, etc.); (3) reliability parameters of distribution network components (the failure rate and mean time to repair of busbars, lines, transformers, circuit breakers, section switches, etc.); (4)load parameters (load points, number of users, etc.) are used as the input feature vector of the deep belief network. The four main reliability indicators of the distribution network: system average interruption 
duration index (SAIDI), system average interruption frequency index (SAIFI), average cumulative outage time (CAIDI) and average service availability index (ASAI) are used as the output feature vector of the deep belief network.

The construction and training process of the deep belief network used for distribution network reliability analysis is as follows:

(1) Determine the structure of the DBN network. Set the number of network layers and the number of nodes in each layer. Through repeated experiments, choose a network structure with 4 hidden layers. The maximum number of nodes in each layer is $2 p * q+q$; , wherein $p$ is the number of network structures of the distribution network sample, $q$ is the amount of data contained in a single sample. The number of neurons in the output layer $\mathrm{Q}=4$.

(2) Enter all the sample data for the reliability analysis of the distribution network. The input data volume is $p \bullet q, 90 \%$ of which are selected as training samples, and the remaining $10 \%$ as test samples.

(3) Optimize the parameters of the DBN network model. Input training samples, train all RBMs in DBN step by step, and get the initial values of DBN network parameters. Then, use BP algorithm to perform reverse optimization training on the parameters obtained through training. Repeatedly optimize the model to reduce the reconstruction error $\mu$ to less than $3 \%$ and thus obtain the required DBN network model. The DBN network training flowchart for the reliability analysis of the distribution network is shown in Figure 2.

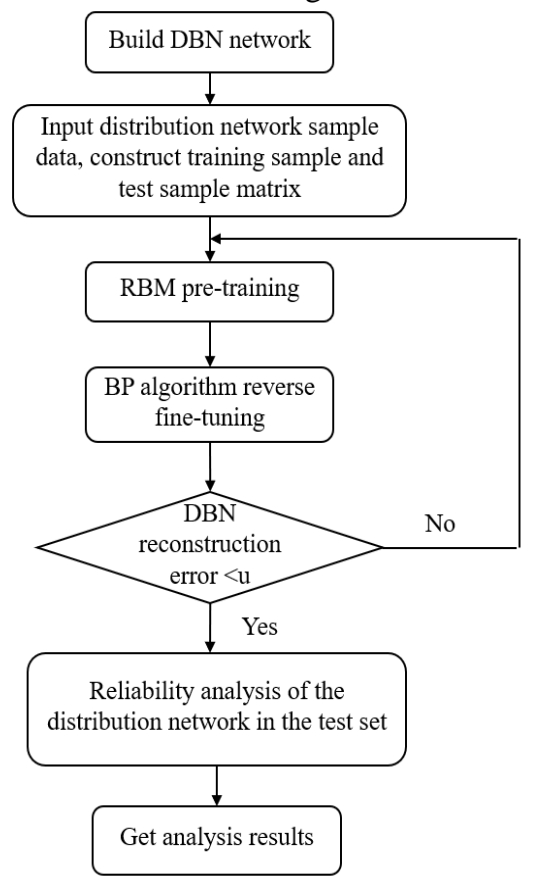

Fig. 2. Flow chart of distribution network reliability analysis based on DBN

\section{Study case}

\subsection{Cases of distribution network reliability analysis based on DBN}

The problem of distribution network reliability analysis is essentially a regression problem, and the DBN model can be used to predict and analyze such problem. This paper uses thousands of distribution network grid samples including hand in hand, ring network, multi-segment single connection, 4 by 6 wiring, radial and other types to train DBN network. This paper chooses $90 \%$ of them as the training set and the remaining $10 \%$ as the test set. As the number of iterations increases during training, the reconstruction error gradually decreases. By repeatedly comparing the errors generated by the DBN network with different parameters, the optimal DBN network parameters are finally obtained as $[312,524,524,524,524,4]$. Input $10 \%$ of the test data into the trained DBN network, and obtain partial test set results based on DBN's SAIFI, CAIDI, SAIDI and ASAI indicators as shown in Figure 3. The abscissa is 100 test set sample points, and the ordinate is the corresponding index.

It can be seen from the figure that the analysis result of DBN is very close to the real value, and the effect is good.

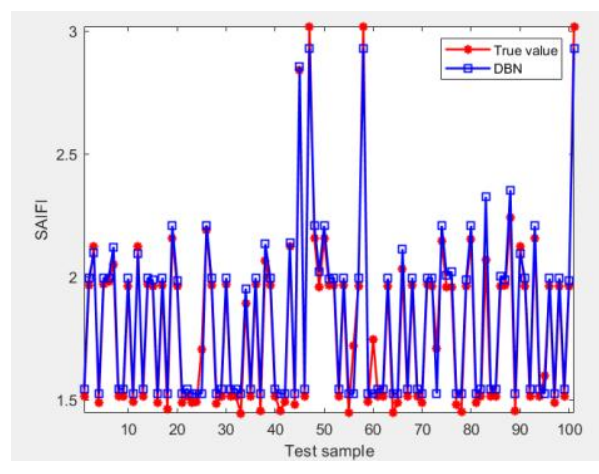

(a) SAIFI

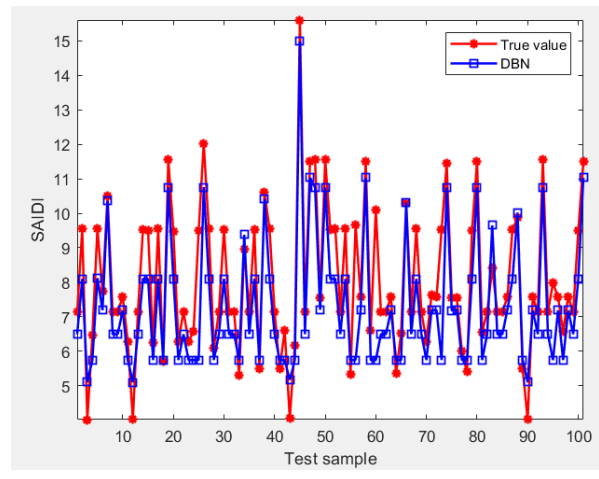

(b) SAIDI

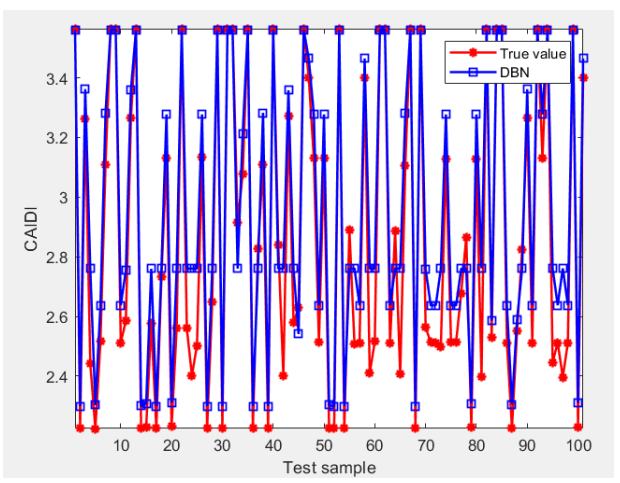

(c) CAIDI 


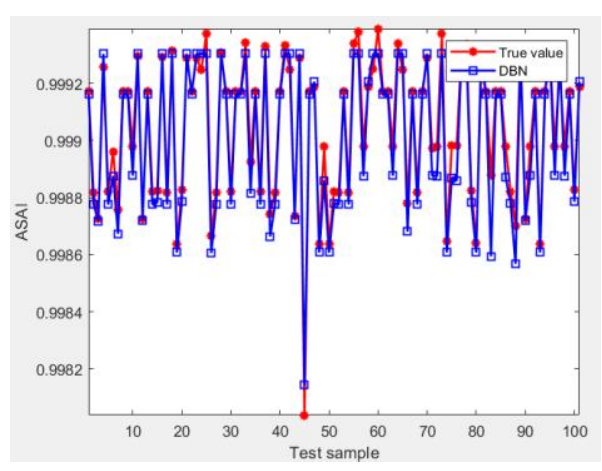

(d) ASAI

Fig. 3. DBN results based on SAIFI, CAIDI, SAIDI and ASAI indicators

\subsection{Error comparison of each model}

The Monte Carlo method in distribution network reliability analysis is an accurate method recognized among traditional methods, and the BP neural network is a representative method of the shallow neural network. In this paper, the distribution network reliability indicators SAIFI, CAIDI, SAIDI and ASAI are used as evaluation indicators. For the same sample, the results of the deep neural network (DBN) are compared with Monte Carlo method and BP neural network method. Root Mean Square Error (RMSE) is selected as the evaluation index of the model. RMSE represents the square root of the deviation between the analysis value and the true value and the square root of the sample ratio, which can better reflect the actual situation of the error. As shown in formula (6).

$$
R M S E=\sqrt{\frac{\sum_{t=1}^{N}\left(w_{f}-w_{t}\right)^{2}}{N}}
$$

Among them, $w_{f}$ is the analysis value of each sample point, and $w_{t}$ is the true value of each sample point. $N$ is the total number of data samples. Using formula (6) to calculate the selected $10 \%$ group of test samples, and the RMSE value obtained based on Monte Carlo, BP neural network, and DBN is shown in Table 1. Choosing one of the indicators (SAIFI) to evaluate the three models. The result of Monte Carlo is 0.93404, the result of $\mathrm{BP}$ is 0.94428 , and the result of $\mathrm{DBN}$ is 0.80398 .

It can be judged that the effect of the DBN model is better than the two models of Monte Carlo and BP.

Table 1. RMSE values based on Monte Carlo, BP, DBN

\begin{tabular}{|c|c|c|c|c|}
\hline & SAIFI & SAIDI & CAIDI & ASAI \\
\hline Monte Carlo & 0.93404 & 0.87542 & 0.95596 & 0.033366 \\
\hline BP & 0.94428 & 0.80791 & 0.94314 & 0.0045537 \\
\hline DBN & 0.80398 & 0.68239 & 0.73848 & 0.0029028 \\
\hline
\end{tabular}

\section{Conclusion}

DBN can fully mine the complex structural laws in data and is widely used to solve regression problems. The method of distribution network reliability analysis based on DBN model proposed in this paper gives full play to the advantages of DBN model. The DBN model used is stacked by restricted Boltzmann machines (RBM). After pre-training all RBMs, the $\mathrm{BP}$ algorithm is used for reverse fine-tuning to complete the training process of the entire network. After the training is completed, the DBN model is used to analyze the test samples, and the analysis results are compared with the analysis results of the BP neural network method and the Monte Carlo simulation method. The results show that the DBN model proposed in this paper has smaller errors and more accurate results, which to a certain extent indicates that the deep learning method has a certain development space in the application field of distribution network reliability analysis.

\section{References}

1. S. Y. Ge, H. M. Wang, L. Xu, Power Sys Techno 36(04), 39-44 (2012)

2. M. D. Gregorio, C. Javier, A. J. M, Transactions on Power Systems 99(1), 95-99(2017)

3. T. Yong, ICCSN 2011 5, 27-29(2011)

4. H. F. Zheng, Z. Q. Huang, Proceedings of the CSU-EPSA 30(04), 144-150(2018)

5. A. Heidari, V. G. Agelidis, IEEE T POWER SYST 33, 412-420(2018)

6. G. E. Hinton, Momentum 9(1), 599-619(2012)

7. G. L. Cai, Y. Lin, J. J. Huan, PMAPS,1-8 (2016)

8. G. Celli, E. Ghiani, F. Pilo, ELECTR POW SYST 104, 164-175(2013)

9. Bae I-S, Kim J-O, IEEE T POWER SYST 23(3), 1416-1422(2008)

10. J. G. Zhou, L.L.Li, Z. G. Li, 6th FSKD 2009, 608-611 (2009)

11. G. E. Hinton, S. Osindero, Y. W. Teh, NEURAL COMPUT 18(7), 1527-1554(2006)

12. G. Munoz-Delgado, J. Contreras, J. M. Arroyo, IEEE T POWER SYST 33(3), 2583-2596(2017)

13. Q. Li, Y. Qiao, Y. J. Zhang, Power Sys Techno, 4(2020)

14. L. Zhang, J. B. Ren, Y. Qiu , M. Jin, electric.power.ict, 12(2019)

15. Z. Y. Li, Journal of Jinling Institute of Technology, 4(2007) 